\title{
基于蒙特卡洛方法的闪炼光纤阵列空间分辨率 模拟
}

\author{
贾清刚 ${ }^{\mathbb{1}} ，$ 王东明 ${ }^{\mathbb{1}}$ ，安洪振 ${ }^{\circledR}$ ，胡华四 $\mathbb{1}^{*}$
}

(1) 西安交通大学核科学与技术学院，西安 710049;

(2) 环境保护部核与辐射安全中心, 北京 100082

*E-mail: huasi_hu@mail.xjtu.edu.cn

收稿日期: 2013-09-10; 接受日期: 2013-10-15

国家自然科学基金(批准号: 10975113)和教育部创新团队计划(批准号: IRT1280)资助项目

\begin{abstract}
摘要阐述塑料闪㷧光纤对 $14.1 \mathrm{MeV}$ 中子的响应机理; 建立基于 Geant4 与 MCNPX 的蒙特卡洛模拟程序, 通过添加发光计算实现 MCNPX 对中子光响应的计算, 并对 Geant4 进行校验; 对 $14.1 \mathrm{MeV}$ 中子在闪㷧体内产生反冲质子及发光响应进行了模拟; 发现并研究了塑料闪㷧内由 $14.1 \mathrm{MeV}$ 中子产生的反冲质子半高宽, 与使用阵列型探测 器获得的半高宽的差异; 给出使用倾斜狭缝源获得的调制传递函数, 结合瑞丽判据获得 精确光纤阵列分辨率的方法; 对正方形光纤阵列与圆柱光纤 (四边形排布与六边形排布) 阵列空间分辨率随光纤尺寸变化进行模拟与分析. 提出的方法与模拟结果可以用于 ICF 实验中光纤阵列的优化设计.
\end{abstract}

关键词

Geant4

闪柏发光

闪炼光纤阵列探测器

空间分辨率
在惯性约束聚变与磁约束聚变等研究中，聚变 核心产生携带重要时空信息的 $14.1 \mathrm{MeV}$ 中子可以穿 透高密度等离子体边界, 利用脉冲中子图像诊断系 统可以获得聚变反应所产生中子强度的空间分布, 根据所获得的反应区对称性、尺寸等信息提供可以 检验相关物理设计与数值模拟计算结果, 并进一步 改进探测系统. 中子探测器是该中子图像诊断系统 的关键部件之一, 其对获得高分辨率的聚变源区图 像至关重要. ICF 实验多采用闪炼光纤探测器获得中 子图像, 随着制作工艺的进步, 阵列中光纤的直径 越来越小以获得更好的空间分辨率 ${ }^{[1 \sim 7]}$. 然而, 由于 反冲质子的影响, 使用细光纤只能在一定程度上提 高空间分辨率. 预期的中子产额与实验布局条件下,
使用更细的光纤其得到的图像信噪比会变差，反而 影响重建分辨率, 在实验中为了提高信噪比会将相 邻多根光纤的输出进行累加 ${ }^{[8]}$, 相当于使用了尺寸 较粗的光纤进行探测. 在开展实验之前, 对光纤阵 列的空间分辨率模拟研究与优化设计工作十分重要. 本文使用 Geant4 程序对塑料闪秌体对 $14.1 \mathrm{MeV}$ 中 子的响应进行模拟, 并利用 MCNPX 及响应发光计 算对 Geant4 结果进行校验. 给出了利用倾斜狭缝源 法评价探测器空间分辨率的方法, 对 3 种类型光纤 阵列(正方形、圆柱四边形排布与六边形排布)中光纤 横截面尺寸对空间分辨率的影响. 本文提出的方法 与模拟结果可以用于 ICF 实验中光纤阵列的优化 设计. 


\section{1 塑料闪伢体对 14.1 MeV 中子的光响应}

$14.1 \mathrm{MeV}$ 聚变中子进入塑料闪伢体, 其可能与 氢核发生 $\mathrm{H}(n, n) \mathrm{H}$ 和 $\mathrm{H}(n, \gamma) \mathrm{D}$ 反应, 以及与碳核发生 ${ }^{12} \mathrm{C}(n, n){ }^{12} \mathrm{C},{ }^{12} \mathrm{C}(n, n \gamma){ }^{12} \mathrm{C},{ }^{12} \mathrm{C}(n, \alpha){ }^{9} \mathrm{Be}$ 和 ${ }^{12} \mathrm{C}\left(n, n^{\prime}\right)$ $3 \alpha$ 反应 ${ }^{[9]}$. 产生的次级带电粒子将会在其径迹上沉 积能量并激发闪㷧光. 虽然从截面上讲 $14.1 \mathrm{MeV}$ 中 子与 ${ }^{12} \mathrm{C}$ 发生作用的截面为 1.5 靶, 是与氢核反应截 面 0.7 靶的两倍 ${ }^{[10]}$. 但从能量传递角度, 入射中子最 多可将 $100 \%$ 的自身能量传递给氢核, 然而传递给 ${ }^{12} \mathrm{C}$ 的最多为 $28 \%$. 除此之外, 由于 ${ }^{12} \mathrm{C}$ 相对氢核重, 发光时由于淬灭效应 ${ }^{[11]}$, 同等的能量沉积, 氢核会产 生更多的闪伢光. 因此, $14.1 \mathrm{MeV}$ 中子与氢核的弹性 散射对于最终光信号的产生起主要作用. 如图 1 所示, 在塑料闪炼体中, 弹性散射产生反冲质子的能量与 散射角之间的关系满足:

$$
E_{P}=E_{0} \cos ^{2} \theta,
$$

其中 $E_{P}$ 为反冲质子能量, $E_{0}$ 入射中子能量, $\theta$ 为反冲 质子散射角. 反冲质子会在其径迹上激发荧光(其他 次级带电粒子也会对光产额有一定贡献, 不过份额 较少 ${ }^{[12]}$, 后续讨论), 通过对发光强度来获得中子通 量分布. $14.1 \mathrm{MeV}$ 中子产生的反冲质子在闪胨体内 的发光特性便直接决定对中子位置探测的精度即空 间分辨率.

\section{2 基于 Geant4 与 MCNPX 的闪炼体对中子 发光响应模拟程序}

为了获得由反冲质子产生相对于弹性散射点的 发光分布, 这里使用 Geant4 软件对 $14.1 \mathrm{MeV}$ 中子在 $1 \mathrm{~cm}$ 厚聚苯乙烯(polystyrene, $\mathrm{C}_{8} \mathrm{H}_{8}, 1.05 \mathrm{~g} / \mathrm{cm}^{3}$ )中的 发光响应进行模拟. 其中中子反应模型使用适合热

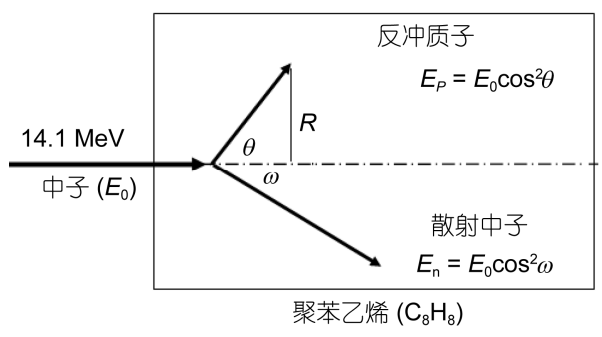

图 1 反冲质子在闪炼体内的产生
中子到 $20 \mathrm{MeV}$ 中子的高精度物理模型(G4Neutron HPElastic, G4NeutronHPInelastic, G4NeutronHPCapture 与 G4NeutronHPFission) $)^{[13]}$. 相应的中子截面来 源于 G4NDL3.13 数据库, 这个数据库的数据主要来 源于 $\mathrm{ENDF} / \mathrm{B}-\mathrm{VI}^{[14]}$. 还使用了标准电磁模型 (EM package, G4EMLOW.6.9 ${ }^{[15]}$. 由于反冲质子等重核的 能量沉积与最终发光并非线性关系, 即淬灭效应, 在 Geant4.9.3 的闪炼发光模块利用半经验的 Birks 公 式 ${ }^{[11]}$ 来描述此非线性关系:

$$
L_{E_{0}}=\int_{0}^{E_{0}} \frac{S \mathrm{~d} E}{1+k B(\mathrm{~d} E / \mathrm{d} x)},
$$

其中 $E_{0}$ 为带电粒子初始能量, $\mathrm{d} E / \mathrm{d} x$ 为该带电粒子 对应能量下的能量损率, $S$ 为常数代表每吸收 $1 \mathrm{MeV}$ 电子能量沉积将产生 8000 个闪炼光子, 经验参数 $k B$ 为 $0.07943 \mathrm{~mm} / \mathrm{MeV}^{[16]}$. 模拟使用了 10 万 $14.1 \mathrm{MeV}$ 中子垂直入射, 产物截断设定为 $0.001 \mathrm{~mm}$. 当反冲 质子降低至不能再产生对应输运长度为 $0.001 \mathrm{~mm}$ 能 量的次级 $\delta$ 粒子时, 对该反冲质子的追踪将按连续能 量衰减模型. 计算闪胨光产额的空间分布时, 仅考虑 由反冲质子的贡献并且没有对光子继续追踪. 将闪 炼光产额分布投影到闪炼平板的后端面, 并利用其 旋转对称特性获得了闪炼发光与散射反应点距离的 关系如图 2 光滑曲线所示. 作为程序校验, 同时使用 $\mathrm{MCNPX}^{[17]}$ 相同模型进行模拟计算. 在 MCNPX 中对 于质子能量沉积计算截断最低为 $1 \mathrm{keV}$, 但是当质子 能量小于 $1 \mathrm{MeV}$ 时, 使用连续能量沉积模型 $\mathrm{d} E / \mathrm{d} x$ 对其进行计算. 由于 MCNPX 仅能计算反冲质子的能 量沉积, 这里同样使用 Birks (2)式对光产额进行计 算. 与 Geant4 计算发光不同的是这里 $\mathrm{d} E / \mathrm{d} x$ 由 SRIM (Stopping and Ranges of Ions in Matter) ${ }^{[18]}$ 获得. MCNPX 经过模拟计算得到闪炼发光与散射反应点 距离的关系如图 2 中点划线所示. 在 MCNPX 中反冲 模拟结果显示, 经由 MCNPX 与发光模型计算的结果 比 Geant4 计算结果稍大, 尤其是在反冲质子径迹的 末端. 这种区别除了积分过程不同外, 还可能是由于 能量截断的方式不同造成的. 从曲线的趋势与结果 来看通过 Geant4 软件可以得到可信的反冲质子发光 分布. 反冲质子在聚苯乙烯中最多能(垂直于中子入 射方向)输运 0.6 0.7 mm. 那么当闪炼光纤半径小于 这个值时, 周围未受辐照光纤必然会输出由反冲质 子产生的串扰光信号. 从探测器本身来讲, 这种情况 


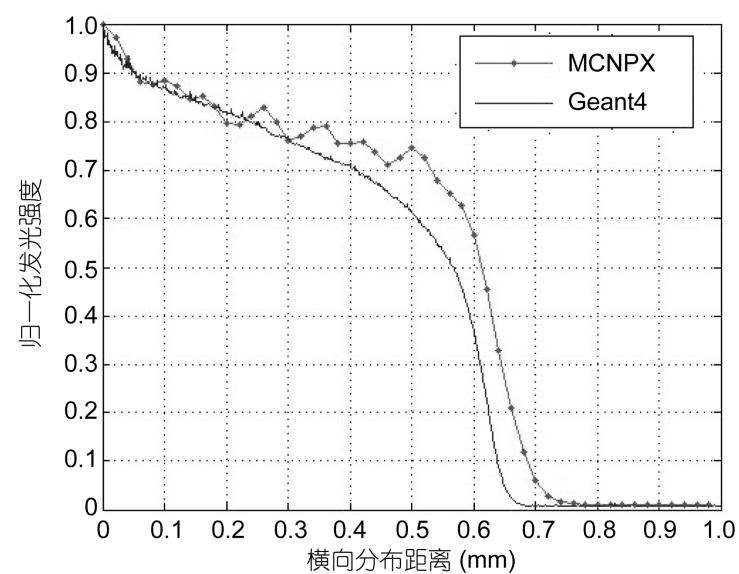

图 2 MCNPX 与 Geant4 模拟获得的在塑料闪㷧体内反冲 质子所产生的发光响应分布

的出现会影响其空间分辨率.

中子在闪㷧体内还有更大的概率与 ${ }^{12} \mathrm{C}$ 作用并 产生光信号, 在模拟中我们首先模拟了仅有反冲质 子作用时的发光分布(峰值归一)如图 3(a)所示, 并模 拟了全部次级带电粒子所产生的光分布 (峰值归一) 如图 3(b) 所示. 图 3 中光滑曲线为发光分布随距离积 分结果. 对比两分布图, 由于其他重带电粒子相比质 子有更短的径迹, 其对发光的贡献主要集中于散射 点附近. 但重带电粒子对于发光的贡献影响 $<10 \%$. 两种光分布的积分结果显示, 无论其他次级粒子是 否参与发光, 距离散射点 $0.25 \mathrm{~mm}$ 范围贡献了约 $50 \%$ 的光产额. 文献 $[2,19]$ 亦给出了相同的结论, 并选择 最佳光纤尺寸(直径或边长)为 $0.5 \mathrm{~mm}^{[19]}$.

\section{3 光纤阵列点扩散函数和线扩散函数的模 拟及分辨率评价方法}

对于光纤阵列的空间分辨率的估计方法常使用 $\sqrt{\left(2 \delta_{\mathrm{pix}}\right)^{2}+\delta_{\mathrm{rec}}^{2}}{ }^{[20]}$ 进行计算, 其中 $\delta_{\mathrm{pix}}$ 为光纤横截面 尺寸, $\delta_{\mathrm{rec}}$ 为反冲质子光响应的半高宽 (FWHM). 前 面获得的 $\delta_{\mathrm{rec}}$ 为 $0.5 \sim 0.7 \mathrm{~mm}$, 那么光纤阵列的空间分 辨率应始终大于中子光响应的半高宽, 但这种评价方 法是有问题的, 这里使用 Geant 4 模拟边长为 $0.02 \mathrm{~mm}$ 的正方形光纤阵列对于 $14.1 \mathrm{MeV}$ 中子的发光点扩散 函数分布. 由于光纤尺寸远小于光响应半径, 得到的 响应半高宽应略大于 $\delta_{\mathrm{rec}}$. 该闪炼光纤类似于圣戈班 的 $\mathrm{BCF}-10$ 系列 ${ }^{[21]}(\mathrm{BCF}-10$ 正方形光纤最小边长为
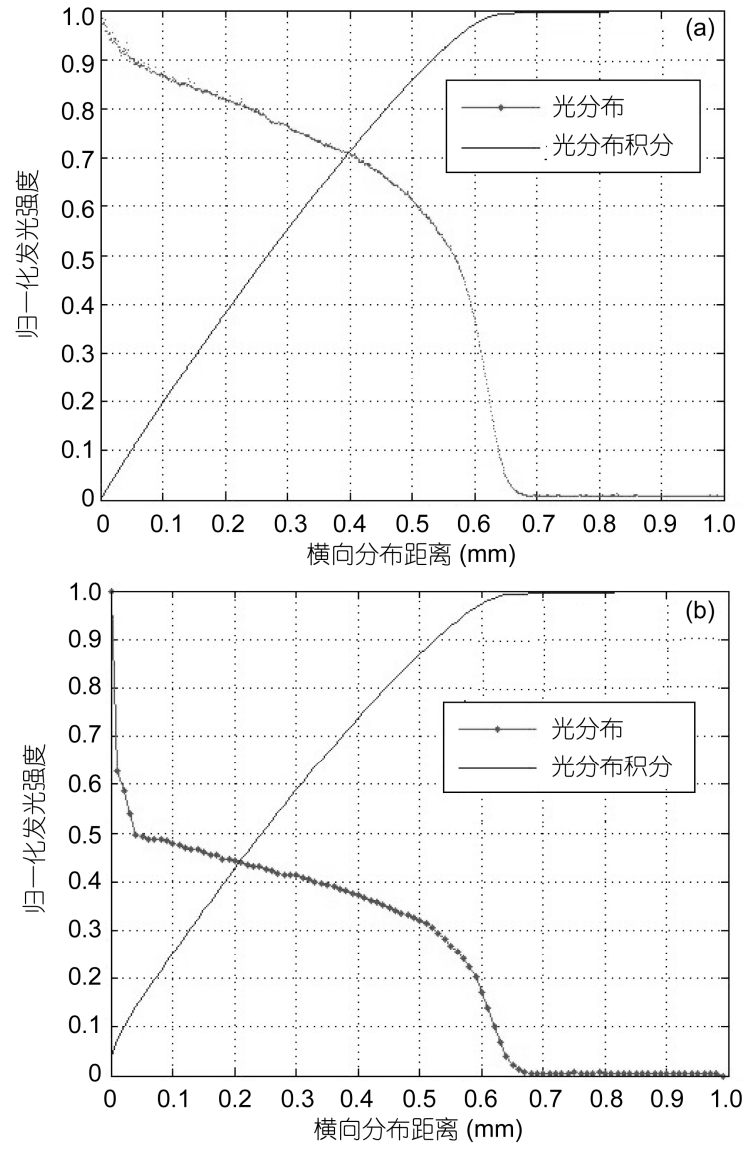

图 3 Geant4 聚苯乙烯塑料闪炼体对 $14.1 \mathrm{MeV}$ 中子发光响 应分布

(a) 仅有反冲质子参与的发光; (b) 所有次级粒子均参与发光

$0.25 \mathrm{~mm})$. 闪炼光纤闪炼芯材料为聚苯乙烯 $\left(\mathrm{C}_{8} \mathrm{H}_{8}\right.$, $1.05 \mathrm{~g} / \mathrm{cm}^{3}$, 折射率为 $1.6,0.018 \mathrm{~mm}$ 边长), 出于建模 考虑，假设此光纤为单反射层光纤，反射层为聚甲基 丙烯酸甲酯 $\left(\mathrm{C}_{8} \mathrm{H}_{5} \mathrm{O}_{2}, 1.2 \mathrm{~g} / \mathrm{cm}^{3}\right.$, 折射率为 $1.49,1 \mu \mathrm{m}$, $0.001 \mathrm{~mm}$ 厚), 闪㐿光在光纤在芯材料与反射层间发 生全反射并传递至光纤端面. 在评价分辨率时常使 用线对的概念, 同时模拟该探测器的线扩散函数. 模 拟获得的点扩散函数与线扩散函数如图 4 所示. 模拟 结果显示, 此类型探测器的点扩散函数及先线扩散 函数半高宽仅有 0.05 与 $0.07 \mathrm{~mm}$, 远小于 $\delta_{\mathrm{rec}}(0.5 \sim$ $0.7 \mathrm{~mm}), \delta_{\mathrm{rec}}$ 这是由于计数方式产生的.

为了阐明计数方式不同对获取的点扩散函数半 高宽的影响, 这里给出了两种计算方式的示意图, 如 图 5 所示. 理想条件下的点扩散函数发光曲线是沿图 5 左半侧白线的光分布, 其获得的点扩散函数半高宽 


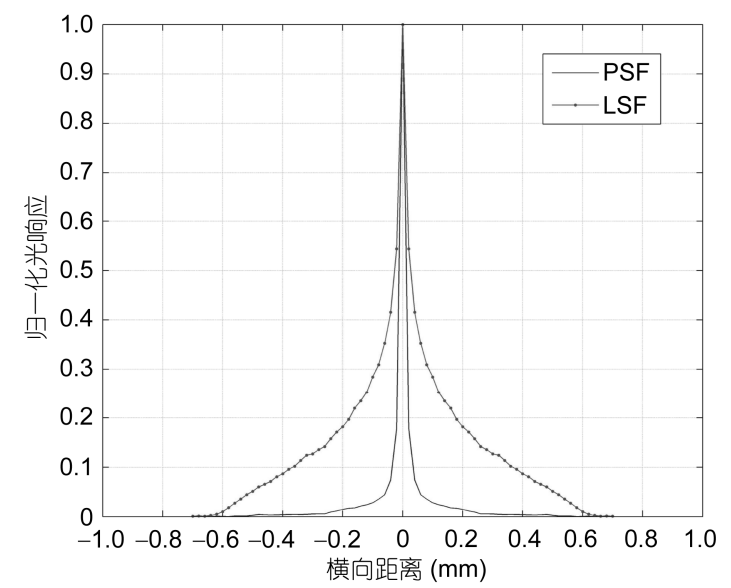

图 $40.02 \mathrm{~mm}$ 边长正方形闪秌光纤阵列对 $14.1 \mathrm{MeV}$ 中子 的光响应截面 (中子源为准直点源与线源)

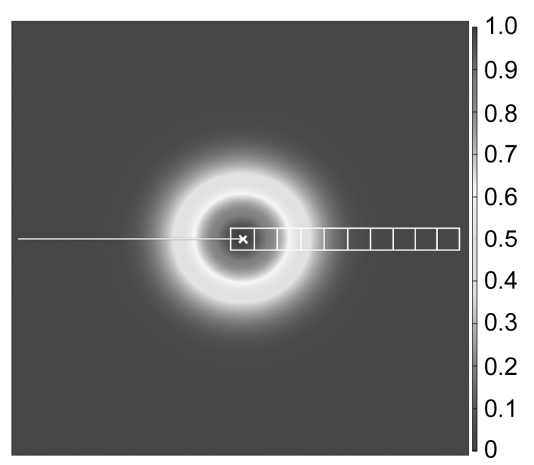

图 5 理想线计数与阵列计数示意图

为 $0.5 \mathrm{~mm}$. 然而, 实际光纤阵列探测器探测的是一 定面积内积分的结果(如图 5 右半侧白色正方形). 按 照文献中公式, 考虑到光纤阵列的离散, 其空间分辨 率应当比闪㷧平板更差. 但如果不考虑实际光纤尺 寸的情况下, 通过单纯计算中子在闪炼体上的发光 响应分布是无法对实际阵列的分辨率进行估计, 更 不能指导光纤半径的优化.

通过蒙特卡洛方法直接模拟探测器对中子的点 扩散函数或者线扩散函数, 利用这些响应函数的半 高宽来评价探测器的分辨率. 然而对于光纤阵列探 测器而言, 中子入射点的位置直接影响到最终光响 应的半高宽. 此外, 当光纤较粗, 如大于 $0.1 \mathrm{~mm}$ 时, 得到的发光响应曲线由几根光纤的光输出数据组成, 其并不连续而是分段函数, 这种情况下只能通过拟 合去获得半高宽. 使用光响应半高宽来评价探测器 分辨率的方法也并不精确, 如图 6 所示, 对于不含统 计噪声的三角形响应函数来说, 半高宽可以描述其

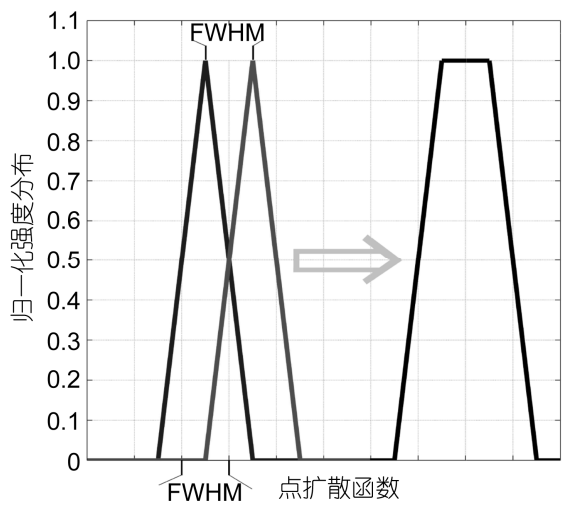

图 6 三角形响应函数及其半高宽(FWHM)

空间分辨率的极限, 然而实际光响应不但含有统计 噪声并且与三角形响应也有一定差距.

为了避免上述问题, 这里倾斜狭缝法 ${ }^{[22]}$ 与瑞丽 判据 ${ }^{[23]}$ 相结合的方法获得探测器空间分辨率. 模拟 时使用倾斜准直线源模拟狭缝, 这样可以避免狭缝 宽度对获得的线扩散函数的影响. 将 $0.1 \mathrm{~mm}$ 边长的 正方形闪炼光纤阵列对于理想倾斜狭缝 $14.1 \mathrm{MeV}$ 中子源光响应(如图 7(a)所示)合成 ${ }^{[22]}$ 为线扩散函数, 如图 7(b)所示. 经由傅里叶变换将线扩散函数转化为 调制传递函数(modulation transfer function, MTF), 如图 7(c) 所示. 再根据瑞丽判据 ${ }^{[23]}$, 当空间频率所对应的 调制传递函数值低于 0.1 时, 空间频率将无法分辨 (对应于调制传递函数值为 0.1 的空间频率即为空间 分辨率).

下面解释调制传递函数值为 0.1 时对应的空间频 率为空间分辨率. 依据瑞丽判据, 两条线能被人眼分 辨的条件是: 两个线(灰度峰值)中间的灰度至少要小 于峰值的 $81 \%$. 对于调制传递函数(MTF)曲线, 分辨 极限或者是最大分辨频率是由 $\mathrm{MTF}=0.1$ 时获得. 下 面通过对调制传递函数的定义公式来说明上述对应 关系, 根据文献[24], 其被定义为

$$
M T F=\frac{M_{\text {captured }}}{M_{o}}=\frac{I c_{\text {max }}-I c_{\text {min }}}{I c_{\text {max }}+I c_{\text {min }}} / \frac{I o_{\text {max }}-I o_{\text {min }}}{I o_{\text {max }}+I o_{\text {min }}},
$$

其中 $M_{\text {captured }}$ 与 $M_{o}$ 分别代表实际探测器获得的调制 传递函数与原始中子通量分布的调制传递函数. $I c_{\text {max }}$ 与 $I c_{\text {min }}$ 分别代表探测器获得图像灰度最高与最 低的强度. $I o_{\text {max }}$ 与 $I o_{\text {min }}$ 代表理想条件下原始中子通 量分布强度的最大与最小值. 当两个线对恰能分辨 时 $I c_{\text {max }}$ 与 $I c_{\text {min }}$ 分别为 1 与 $0.81, I o_{\text {max }}$ 与 $I o_{\text {min }}$ 分别 

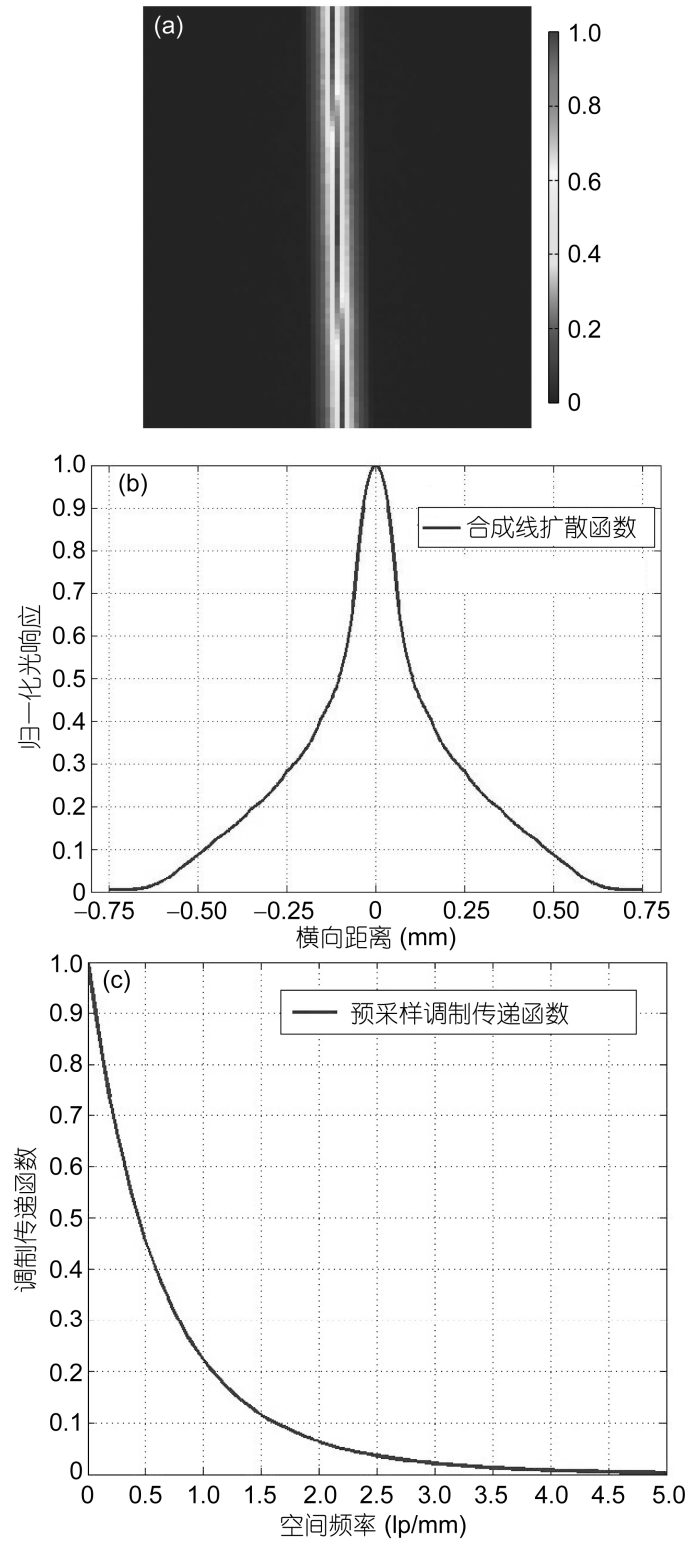

图 7 倾斜狭缝法获得探测器调制传递函数

(a) 光纤阵列对倾斜狭缝中子的光响应; (b) 经光响应合成的线扩散 函数; (c) 由合成线扩散函数径傅里叶变换得到的调制传递函数

为 1 和 0 . 此时, 通过(3)式计算获得的 MTF 为 0.1 , 此 探测器所能分辨的最高频率为 MTF 降低至 0.1 时 的频率. 对于边长为 $0.1 \mathrm{~mm}$ 正方形光纤阵列, 其对 $14.1 \mathrm{MeV}$ 中子的空间分辨率为 1.7 线对每毫米 $(\mathrm{lp} / \mathrm{mm})$. 直观起见, 通过 Geant4 程序模拟该光纤阵 列探测器对二值线对源的响应(如图 8), 空间频率大 于 $1.9 \mathrm{lp} / \mathrm{mm}$ 的线对几乎无法分辨, 而 $1.7 \mathrm{lp} / \mathrm{mm}$ 线对 较容易分辨. 当然人眼对于线对分辨的判断随线对
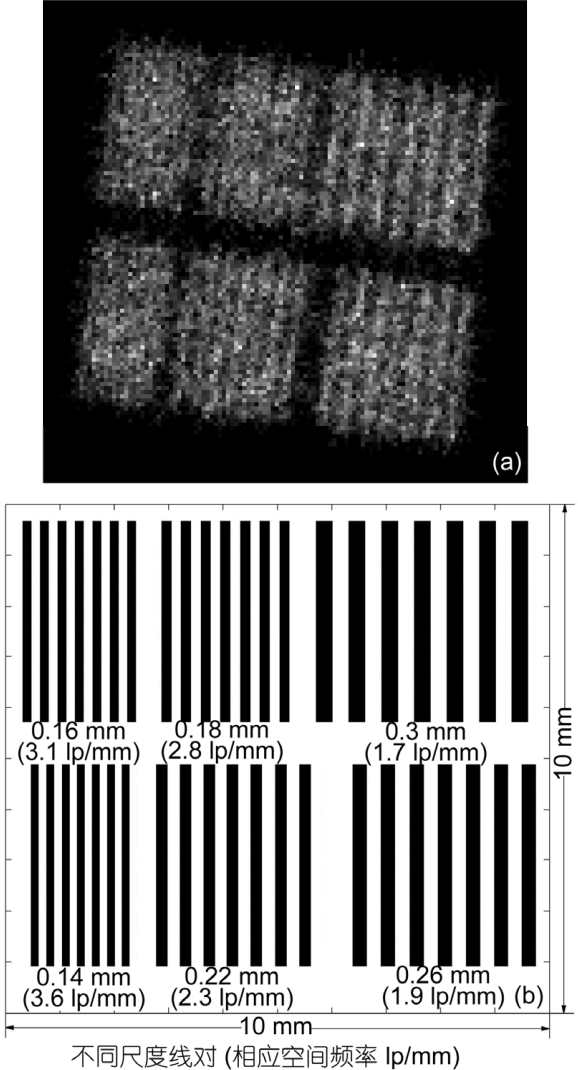

图 $80.1 \mathrm{~mm}$ 正方形光纤阵列所获得的线对源发光图像(每 根光纤垂直入射 300 个中子)(a)与线对中子源示意图(b)

空间频率变化是渐变的, 但通过对线对可以说明该 评价方法较为可靠.

\section{3 种光线阵列空间分辨率模拟}

根据上述分辨率评价方法, 针对常见类型的光 纤阵列研究光纤横截面尺寸与光纤空间分辨率之间 的关系. 常见类型光纤阵列如图 9 所示, 包含以下 3 种: 正方形光纤阵列, 四边形排布圆柱形光纤阵列与 六边形排布的圆柱光纤阵列.

图 10 给出的是正方形光纤阵列的调制传递函数 随光纤边长变化的情况, 其中 $0.05 \mathrm{~mm}$ 正方形光纤阵 列 MTF 计算结果与文献[24]结果吻合. 根据奈奎斯 特采样定律，阵列型探测器的最高采样频率受制于 像素尺寸即光纤横截尺寸. 所以 MTF 曲线的频域范围 均存在截断上限 $(1 /(2 \times$ 像素尺寸 $))$. 例如, 对于 $0.5 \mathrm{~mm}$ 正方形光纤阵列的理论最大(不考虑光纤中质子串扰 对分辨率的影响)分辨率为 $1 \mathrm{lp} / \mathrm{mm}$, 但由于反冲质子 

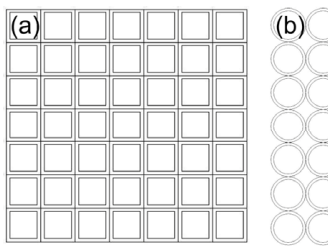

(b)

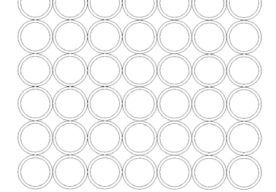

(c)

图 93 种不同类型光纤阵列示意图

(a) 正方形; (b) 圆柱四边形排布; (c) 圆柱六边形排布

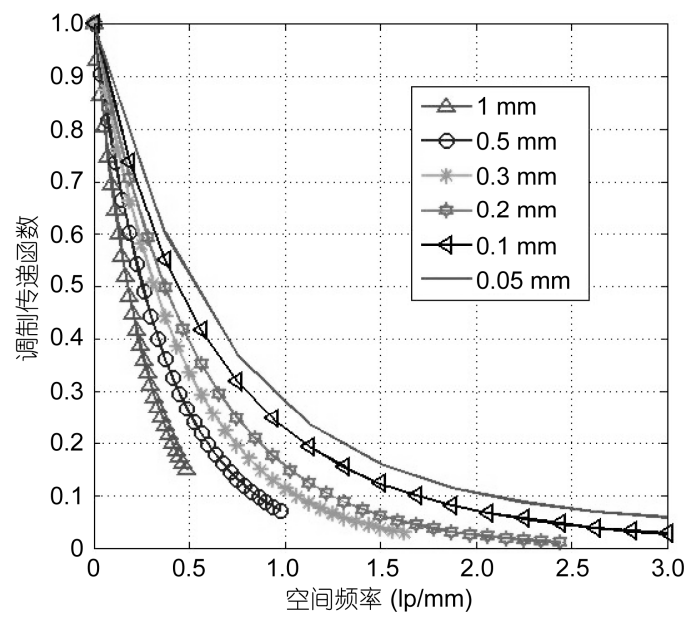

图 10 正方形光纤阵列调制传递函与光纤边长的关系

造成空间分辨率下降, 根据瑞丽判据, 该探测器实际 分辨只有 $0.8 \mathrm{lp} / \mathrm{mm} .1 \mathrm{~mm}$ 正方形光纤阵列的 MTF 始 终大于 0.1 , 在这种情况下采样定律决定该阵列的空 间分辨率.

模拟结果显示, 选用细光纤可以提高空间探测系 统的空间分辨率, 但是当光纤尺寸变细时(小于 $0.7 \mathrm{~mm}$ ), 由于反冲质子影响, 单纯使用细光纤无法有效提高 空间分辨率. 为了说明这个问题, 图 11 给出了无反 冲质子影响时 3 种光纤阵列(正方形、圆柱六边形排 布与四边形排布)的理想分辨 (即可分辨的最高频率 $1 /(2 \times$ 像素尺寸 $))$ 与实际获得的分辨率. 对比细光纤所 能带来的理论分辨与实际分辨率的偏差, 当光纤越 细, 反冲质子对分辨率的影响便越大, 减小光纤尺寸 对分辨率提高的能力便越小. 当光纤尺寸由 $1 \mathrm{~mm}$ 缩减 10 倍至 $0.1 \mathrm{~mm}$ 时, 分辨率可提升 3 倍左右(由 $0.5 \mathrm{lp} / \mathrm{mm}$ 提升至约 $1.5 \mathrm{lp} / \mathrm{mm}$ ). 当使用更细的光纤 $0.05 \mathrm{~mm}$ (替代 $0.1 \mathrm{~mm}$ ) 时, 虽然分辨率仍有一定提升, 但制作工艺难度与成本均将大幅度增加. 同为四边形 排布, 圆柱型光纤比正方形光纤在尺寸为 $0.2 \sim 0.7 \mathrm{~mm}$ 有较好的分辨率, 这是由于圆柱形光纤阵列相比正

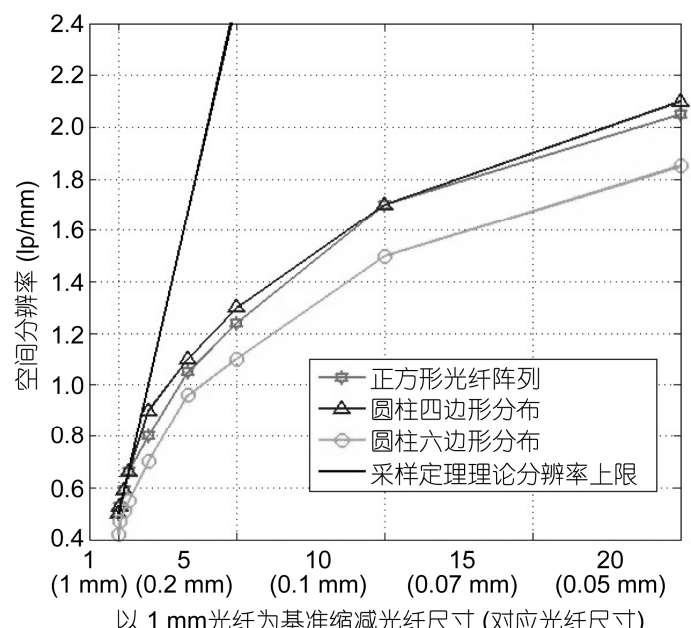

以 $1 \mathrm{~mm}$ 光纤为基准缩减光纤尺寸 (对应光纤尺寸)

图 113 种类型光纤阵列空间分辨率与光纤横截面尺寸(边 长或直径)的关系

方形光纤阵列, 具有更多的死区域. 填充死区域的光 学胶吸收了一部分反冲质子, 减少了串扰的发生. 然 而当光纤较细小于 $0.2 \mathrm{~mm}$ 时, 死区域不能有效阻挡 反冲质子的串扰, 这时两种类型探测器的空间分辨 率偏差不大. 对于紧凑分布的六边形排布圆柱光纤 阵列, 由于光纤之间平均距离小于同样的光纤四边 形排列时, 反冲质子更容易从受辐射光纤输运至邻 近光纤, 使其空间分辨率相对四边形分布较差.

\section{5 结论与展望}

理论计算方法对光纤阵列的空间分辨率的估计 可能会带来很大误差. 使用点扩散函数描述光纤阵 列空间分辨率时，模拟点扩散函数的连续性会受到 光纤尺寸的制约, 并且中子入射点的选择也会影响 获得的结果.

本文使用两种蒙特卡洛程序 Geant4 与 MCNPX 对闪炼光纤对中子的光响应及所产生的反冲质子的 特性进行了模拟. 利用倾斜狭缝获得连续的合成线 扩散响应函数并计算出探测器的调制传递函数的方 法用以评价探测器空间响应. 模拟结果表明, 实际阵 列的空间分辨率并非如理论预期的大于反冲质子光 响应半高宽 $(0.5 \mathrm{~mm})$, 而可能远小于 $0.5 \mathrm{~mm}$. 圆柱光 纤四边形排列与相同横截面特征尺寸的正方形光纤 的空间分辨率基本一致(0.1 0.7 $\mathrm{mm}$ 范围内略好). 对 于六边形排布圆柱光纤阵列由于其排列较为紧凑, 受的反冲质子影响更大, 空间分辨率比另外两类阵 
列较差.

本文提出的方法与模拟结果可以用于 ICF 实验 中光纤阵列的优化设计. 在预期的中子产额与实验

布局条件下, 使用过细的光纤所得到的图像信噪比
较差, 反而影响重建分辨率. 考虑到细光纤阵列的成 本问题，在开展实验之前，应结合具体实验条件，如 成像编码孔、放大倍数、预期中子产额等, 利用蒙卡 方法对光纤阵列探测器进行优化设计.

\section{参考文献}

1 Ress D, Lerche R A, Ellis R J, et al. Design of a neutron penumbral-aperture microscope with $10 \mu \mathrm{m}$ resolution. Rev Sci Instrum, 1990, 61: 3184-3186

2 Disdier L, Lerche R A, Bourgade J L, et al. Capillary detector with deuterated scintillator for inertial confinement fusion neutron images. Rev Sci Instrum, 2004, 75: 2134-2139

3 Ress D, Lerche R A, Ellis R J, et al. High-sensitivity scintillating fiber imaging detector for high-energy neutrons. Rev Sci Instrum, 1995, 61: 4943-4948

4 Wilke M D, Batha S H, Bradley P A, et al. The national ignition facility neutron imaging system. Rev Sci Instrum, 2008, 79: 10E529(1-5)

5 Caillaud T, Landoas O, Briat M, et al. A new compact, high sensitivity neutron imaging system. Rev Sci Instrum, 2012, 83: 10E131(1-3)

6 Disdier L, Rouyer A, Lantuejoul I, et al. Inertial confinement fusion neutron images. Phys Plasmas, 2006, 13: 056317(1-6)

7 Disdier L, Rouyer A, Wilson D C, et al. High-resolution neutron imaging of laser imploded DT targets. Nucl Instrum Meth Phy Res A, 2002, 489: 496-502

8 Knoll G F. Radiation Detection and Measurement. New York: Wiley, 2010. 569-579

9 IAEA. Nuclear Data Services. Available: https://www-nds.iaea.org/exfor/endf.htm

10 Briks J B. The Theory and Practice of Scintillation Counting. Oxford: Macmillan, 1964, 8-10

11 UCRL-50176. Detector Handbook. Lawrence Livermore Lab, 1967

12 CERN. Geant4 User support. http://geant4.web.cern.ch/geant4/UserDocumentation/UsersGuides/PhysicsReferenceManual/fo/PhysicsReferenceManual.pdf

13 Allison J, Amako K, Apostolakis J, et al. Geant4 developments and applications. IEEE Trans Nucl Sci, 2006, 53: 270-278

14 Burkhardt H, Grichine V M, Gumplinger P, et al. Geant4 standard electromagnetic package for HEP applications. In: Proceedings of the 2005 IEEE International Conference on Nuclear Science. Rome, 2004. 1907-1910

15 Angela L T. "HCAL reconstruction status," presented at the Calice Collaboration Meet. Argonne, IL, USA, March 17-19, 2008. http://www.desy.de/ lucaci/Talks/Argonne_March08.pdf

16 MCNPX User's Manual. Los Alamos Nat Lab. Report LA-CP-02-408, April, 2002

17 Ziegler J F, Biersack J P, Littmark U. SRIM-The stopping and range of ions in matter (2010). Nucl Instrum Meth Phy Res B, 2010, 268: $1818-1823$

18 Mor I, Vartsky D, Feldman G, et al. Parameters affecting image quality with Time-Resolved Optical Integrative Neutron (TRION) detector. Nucl Instrum Meth Phy Res A, 2011, 640: 192-199

19 Disdier L, Rouyer A, Fedotoff A, et al. Neutron imaging of ICF target plasmas. Rev Sci Instrum, 2003, 74: 1832-1836

20 Brochure of Saint-Gobain Scintillation Products. Saint Gobain Crystals and Detectors Ltd. Hiram, OH. USA. http://www.detectors.saintgobain.com/uploadedFiles/SGdetectors/Documents/Brochures/Scintillating-Optical-Fibers-Brochure.pdf

21 Fujita H, Tsai D Y, Itoh T, et al. A simple method for determining the modulation transfer function in digital radiography. IEEE Trans Med Imag, 1992, 11: 34-39

22 Dekker A J, Bos A. Resolution: A survey. Jour Opt Soc Am A, 1997, 14: 547-557

23 Boreman G D. Modulation Transfer Function in Optical and Electro Optical Systems. Bellingham: SPIE Press, 2001. 20-25

24 Vartsky D, Mor I, Goldberg M B, et al. Time-resolved fast neutron imaging: Simulation of detector performance. Nucl Instrum Meth Phy Res A, 2005, 542: 206-212 